\title{
Variation Between Observers in Describing Mucosal Appearances in Proctocolitis*
}

\author{
J. H. BARON, $\dagger$ M.A., B.M., M.R.C.P.; A. M. CONNELL, $\ddagger$ M.B., B.SC.; \\ J. E. LENNARD-JONES, $§$ M.A., M.B., M.R.C.P.
}

Brit. med. Y, 1964, 1, 89-92

The appearances of the recto-sigmoid mucosa in proctocolitis are so diverse that it is difficult to establish generally applicable and precisely defined descriptive terms. As these appearances are used in diagnosis and as an indication of the course of the disease, it is important that the overall visual impression should be analysed into its various components and that the observervariation in describing them should be known.

We have made such an analysis and measured the observervariation in describing each component. As might be expected, we have found that observer-variation for graded characteristics -for example, shades of red-is high and that for the few discontinuous variables-for example, the presence or absence of blood in the lumen-it is low. Using the best of these criteria, it may be possible to describe mucosal appearances in a more consistent way.

The study has been limited to the mucosal appearances seen at sigmoidoscopy with and without a telescope. No attempt has been made to correlate these appearances with either the clinical course of proctocolitis or with the histological appearances of the mucosa inspected. No claim is made that the characteristics defined necessarily have any relation to the severity of the disease. We have analysed observations recorded by three observers at one time and under the same conditions ; but we have not devised a method of investigating the consistency with which one observer records at different times.

\section{Conduct of Trial}

The trial was carried out in a medical clinic at which 40-50 patients with colitis are seen each week. During the previous 20 months, while we had been working together as a team, each had performed about a thousand sigmoidoscopies on patients with colitis, and the appearances observed had often been discussed between us.

The 60 patients examined during this trial were taken at random from those attending the clinic, and they agreed to a longer examination than usual. The order in which the three observers examined the patients was varied according to a Latin-square pattern. The patients had no special bowel preparation and lay in the left lateral position. A Lloyd-Davies $25-\mathrm{cm}$. sigmoidoscope, $13 \mathrm{~mm}$. bore $\times 1.5$ eyepiece (or $\times 3$ telescope) was inserted by the first observer, and each in turn examined the appearances and recorded them independently on special forms.

\section{Scoring of Results}

Where a choice of three or more adjectives is available to each of three observers the following results are possible-agreement, dissent (used in the sense of a minority view), and disagreement

\footnotetext{
- From St. Mark's Hospital. London.

† Lately Leverhulme Research Scholar, Institute of Clinical Research, Middlesex Hospital Medical School, London.

¥ Memher of the Medical Research Council Gastroenterology Research Unit, Central Middlesex Hospital, London.

8 Lately Senior Registrar, Department of Gastroenterology, Central Middlesex Hospital, Zondon.
}

(all observers differ). Thus, in assessing the colour of the mucosa three adjectives might be available-red, pink, paleso that three results are possible-agreement, dissent, and disagreement (Fig. 1).

\begin{tabular}{|c|c|c|c|}
\hline A & B & C & RESULT \\
\hline PINK & PINK & PINK & AGREEMENT \\
\hline PINK & RED & RED & \% DISSENT \\
\hline PINK & RED & PALE & DISAGREEMENT \\
\hline
\end{tabular}

FIG. 1.-Possible results when three observers have to choose one of three or more adjectives.

Where a choice of two adjectives is available to each of three observers the results are limited to the alternatives agreement and dissent (disagreement is impossible), as in assessing friability with only two possibilities, friable and not friable (Fig. 2).

\begin{tabular}{|c|c|c|c|}
\hline A & B & C & RESULT \\
\hline FRIABLE & FRIABLE & FRIABLE & FAGREEMENT \\
\hline FRIABLE & NOT FRIABLE & FRIABLE & \% OISSENT: \\
\hline NOT FRIABLE & NOT FRIABLE & FRIABLE & OISSENT:
\end{tabular}
FIG. 2.-Possible results when three observers have to choose one
of two adjectives.

Observer-variation is likely to be greater when three adjectives are available for description than when two adjectives are available. We have therefore considered the criteria in which two choices were available separately from those in which three choices were available to the observers.

\section{Patients 1-30}

\section{Procedure}

In the first 30 patients 13 criteria were selected (Table I), and the observers examined the mucosa with respect to each

\footnotetext{
TABLE I.-Criteria, and Choice of Adjectives Available for Describing Them, Used in Patients 1-30$$
\text { Wall }
$$$$
\text { A. Colout : normal pink; red; very pale }
$$$$
\text { B. Laige deep vessels: normal; none; extra }
$$$$
\text { Superficial small vessels : normal few ; none ; many }
$$$$
\text { Moisture : normal dry; moist/oedematous }
$$$$
\text { Friability: normal non-friability ; friable }
$$$$
\text { Granularity : normal smooth; granular }
$$$$
\text { Distensibility : normal distensibility ; rigid/contracted }
$$$$
\text { Polyp : smooth mucosa ; polyp/pseudopolyp }
$$$$
\text { Lumen }
$$$$
\text { J. Blood : no blood; blood }
$$$$
\text { Lumen }
$$$$
\text { Grading of "Activity" }
$$

Normal : pale with vascular pattern. Inactive : dry, granular, not friable. Moderate activity : moist, granular, slightly friable.
Active : moist, friable, not granular.
} 
criterion and chose one of the possible adjectives given. The mucosa was examined at a distance of $10 \mathrm{~cm}$. from the anus in Patients 1-10 and at a distance of $15 \mathrm{~cm}$. in Patients 11-20. Inspection at these arbitrary distances occasionally resulted in the placing of the sigmoidoscope at positions where slight movement caused marked changes in the appearances of the mucosa, so that in Patients 21-30 the sigmoidoscopic appearances were noted at a level selected by the first observer as being an area where the appearances were reasonably uniform over a few centimetres. In Patients 11-30 the mucosa was inspected first with the $\times 1.5$ eyepiece and then with a $\times 3$ telescope. Any changes in the assessment of blood vessels, granularity, ulceration, and "activity" with the higher magnification were noted.

\section{Results}

Fig. 3 sets out the results for criteria in which a choice of two adjectives was available. All the criteria except granularity showed $60 \%$ agreement or more ; the use of the telescope did not increase the measure of agreement for granularity. The

\begin{tabular}{|c|c|c|c|c|c|}
\hline & & & $\begin{array}{c}\text { AGREEMENT } \\
18\end{array}$ & 1 & $\begin{array}{c}\text { DISSENT } \\
12\end{array}$ \\
\hline \multirow{2}{*}{ MOISTURE } & DRY & MOIST & & 然 & \\
\hline & & & 22 & 1 & 8 \\
\hline \multirow{2}{*}{ FRIABILITY NC } & ON-FRIABLE & FRIABLE & & a & \\
\hline & & & 14 & 1 & 16 \\
\hline \multirow[t]{2}{*}{ GRANULARITY } & SMOOTH & GRANULAR & -2 & $y$ & \\
\hline & & & 21 & 1 & 9 \\
\hline \multirow[t]{2}{*}{$\begin{array}{c}\text { BLOOD IN } \\
\text { LUMEN }\end{array}$} & PRESENT & ABSENT & & -2 & \% \\
\hline & & & 26 & 1 & 4 \\
\hline \multirow[t]{2}{*}{ DISTENSIBILITY } & NORMAL & $A \mid G 10$ & ma & $x_{2}$ & \\
\hline & & & 30 & 1 & \\
\hline \multirow[t]{2}{*}{ POLYP } & PRESENT & ABSENT & & 1 & z) \\
\hline & & & 30 & 1 & \\
\hline \multirow[t]{2}{*}{ ULCER } & PRESENT & ABSENT & 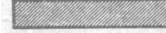 & -12 & \\
\hline & & & $\begin{array}{r}60 \% \\
\text { L AGREEME }\end{array}$ & I & \\
\hline
\end{tabular}

FIG. 3.-Results for two-choice criteria in Patients 1-30.

\section{Patients 31-60}

\section{Procedure}

In the light of the experience with the first 30 patients new criteria were used to describe the appearance in a second group of 30 patients. Eight criteria were used (Table II) and one adjective was selected to describe each of them as before. The following changes were made: (1) The appearance of bloodvessels was more closely defined as superficial and ramifying,

TABLE II.-Criteria, and Choice of Adjectives Available for Describing Them, Used in Patients 31-60.

$$
\text { Wall }
$$

A. Superficial ramifying blood vessels : normal throughout; patchy; none B. Spontaneous bleeding ahead of instrument: none; presen

C. Bleeding to light touch (friability): none; present

E. Mucosal surface : normal mat ; dull, lustreless ; wet and shiny

F. Ulceration : no ulcers; ulcers

$$
\text { Grading of "Activity" }
$$

(0) Normal : mat mucosa, ramifying vascular pattern clearly visible throughout, no spontaneous bleeding, no bleeding to light touch.

(1) Abnormal but not haemurrhagic : appearances between $(0)$ and (2). Moderately haemnrrhagic: bleeding to light touch, but no spontaneous bleeding seen ahead of instrument on initial inspection

(3) Severely hoemorrhagic: snontaneous bleeding seen ahead of instrument at initial inspection, and bleeds to light touch

and the use of a new adjective, patchy, in the description of these vessels was investigated. (2) We differentiated between spontaneous bleeding seen ahead of the instrument and bleeding to light touch with the tip of the sigmoidoscope (friability) (3) An attempt was made to define the sheen on the mucosal surface in photographic terms, and this description was tested. (4) A new criterion, the appearance of the valves, was introduced. (5) We revised the grading of "activity" on the basis of mucosal bleeding and the presence or absence of a ramifying vascular pattern, omitting granularity. (6) Some poorly reproducible characteristics (colour, small vessels, mucopus, faeces) and two characteristics noted by only one observer (distensibility and extra blood vessels) were omitted.

The sigmoidoscopic appearances in Patients 31-60 were noted at a level selected by the first observer as being an area which

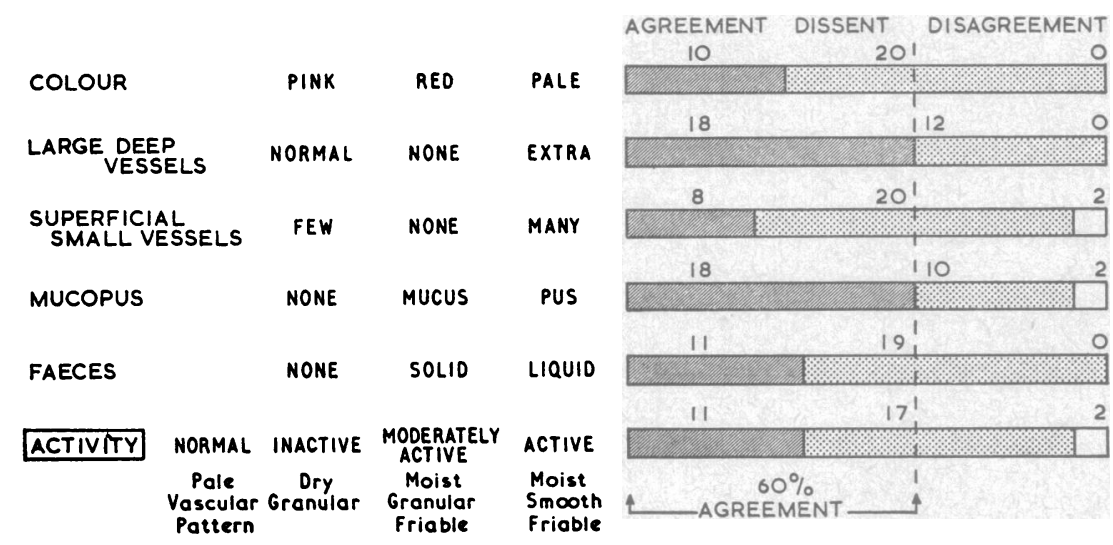

FIG. 4.-Results for criteria with three or more choices in Patients 1-30. characteristic " distensibility" showed a high degree of agreement because only one observer recorded any results as rigid or contracted. The characteristics "polyps" and "ulcers" showed absolute agreement because none were seen.

Fig. 4 sets out the results for criteria for which a choice of three or more adjectives was available. No criteria except the description of large blood-vessels showed $60 \%$ agreement and/ or no disagreement.

There was agreement in the final grading of "activity" in only 11 of 30 patients, there was dissent in 17 , and disagreement in 2. The use of the telescope did not improve the results for any of the criteria in which it was used and the results did not improve as the trial proceeded. included a valve and where the appearances were reasonably uniform over a few centimetres. He checked that the appearances were as he had recorded them before the second and third observers recorded their findings. Care was taken to hold the sigmoidoscope at one level throughout the examination. The characteristics "granularity" and "ulcers" and the grading of "activity" were assessed with the telescope as well as the ordinary eyepiece.

\section{Results}

The results for criteria in which a choice of two adjectives was available are set out in Fig. 5. All criteria except granu- 
larity showed more than $60 \%$ agreement; again the use of the telescope did not improve the result for this criterion. Using the definitions "spontaneous bleeding" as that seen ahead of the instrument and "friability" as bleeding to light touch with the tip of the sigmoidoscope, agreement was about $90 \%$.

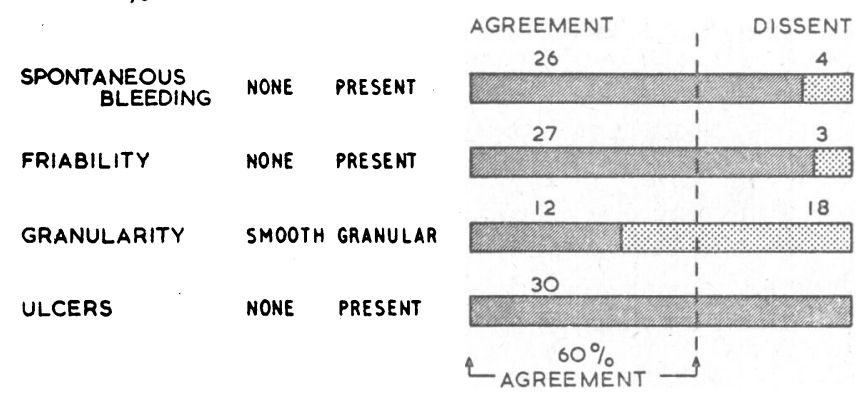

Fig. 5.-Results for two-choice criteria in Patients 31-60. found to be patches of mucus that can be moved with the tip of the instrument.

There was no trend towards the recording of different appearances on the second or third of the three occasions of study, so that the presence of the sigmoidoscope in the lumen of the bowel throughout the period of examination did not apparently alter the appearances of the bowel ahead of the instrument. One of the observers recorded granularity more of ten than either of the other two, and this difference accounts for some of the variability in describing this characteristic.

In treatment trials at this clinic (Baron et al., 1962) the sigmoidoscopic appearances are assessed by two observers. The agreement in their assessment of "activity" reached by the three observers in this trial, taken in pairs, is shown in Table III. It will be seen that in Patients 1-30 agreement was reached in only about one-half, whereas in Patients $31-60$, using the revised grading of " activity," agreement between two observers was uniformly reached on two occasions out of three.

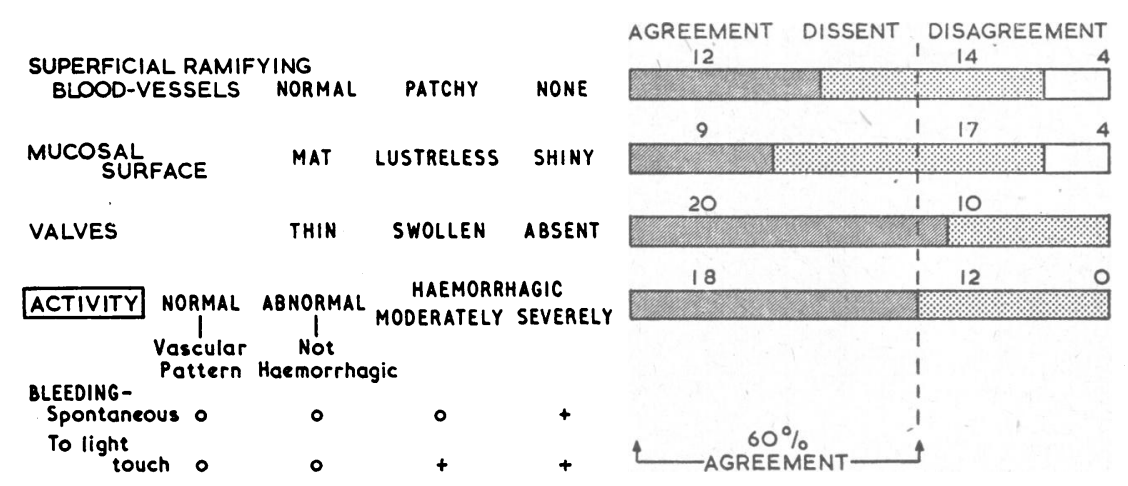

FIG. 6.-Results for criteria with three or more choices in Patients $31-60$.

Fig. 6 sets out results for criteria in which a choice of three or more adjectives was available. The adjective "patchy" in the assessment of the superficial ramifying blood-vessels was not helpful, as it reduced agreement to $40 \%$ and introduced disagreement. The assessment of the mucosal surface, using the photographic terms, was poorer than with the two simple adjectives, dry and moist, used in the first part. The description of the valves showed $60 \%$ agreement and no disagreement. Using the new grading of " activity," the three observers agreed in 18 of the 30 patients and there was no disagreement. The result was similar when the telescope was used. Possibly the most important distinction is between non-haemorrhagic and haemorrhagic, using the term "haemorrhagic" to include both friability and spontaneous bleeding, and agreement on this was reached in 26 out of 30 patients. The agreement in grading of "activity" apparently improved as the second part of the trial proceeded, being 3 out of 10,6 out of 10 , and 9 out of 10 in successive groups.

\section{Results Common to All Patients}

A few characteristics were common to both parts of this study. The observers agreed in their assessment of friability in 49 of the 60 patients but in their assessment of granularity in only 26 of the 60 .

No macroscopic ulcer was seen by any of the observers, with or without the telescope, in any of the 60 patients. This may seem surprising in a disease termed " ulcerative colitis." Ulcers may not have been seen because this trial was conducted in an out-patient clinic and so patients with severe colitis were not seen. However, even in patients with severe disease, LockhartMummery (1959) has shown in operation specimens that macroscopic ulcers, though common in the colon, are rare in the rectum. White patches over the mucosa at sigmoidoscopy are sometimes thought to be ulcers, but these are almost invariably
TABLE III.-Agreement Between Pairs of the Three Observers in Grading the "Activity" of the Rectosigmoid Mucosa. The Classification of "Activity" Used in Patients 1-30 Differed from that Used in Patients 31-60

\begin{tabular}{|c|c|c|c|c|c|}
\hline & & & \multicolumn{3}{|c|}{ Observers } \\
\hline & & & 1 and 2 & 1 and 3 & 2 and 3 \\
\hline Patients $1-30$ & $\left\{\begin{array}{l}\text { Agreement } \\
\text { Disagreement }\end{array}\right.$ & .. & $\begin{array}{l}15 \\
15\end{array}$ & $\begin{array}{r}21 \\
9\end{array}$ & $\begin{array}{l}14 \\
16\end{array}$ \\
\hline$\Rightarrow \quad 31-60$ & $\left\{\begin{array}{l}\text { Agreement ... } \\
\text { Disagreement }\end{array}\right.$ & $\ldots$ & $23(24)$ & $\begin{array}{r}22(22) \\
8(8)\end{array}$ & $21(22)$ \\
\hline
\end{tabular}

Numbers in parentheses are results when the telescope was used.

\section{Conclusions}

We have shown that the characteristics which can be used with some confidence in describing mucosal appearances are those in which the observer records the presence or absence of a clearly defined feature. In this category of discontinuous variables are the occurrence of spontaneous bleeding, bleeding to light touch (friability), and the presence or absence of ramifying blood-vessels. Good agreement can also be obtained if a continuum is divided into its two extremes-for example, dry or moist. "Granularity" does not follow this rule, perhaps because the term is used to describe an exaggeration of the normal unevenness of the mucosal surface.

Of little use as descriptive criteria are those in which the observer has to discriminate between poorly defined parts of a continuum, such as shades of colour, or whether blood-vessels are visible throughout or only in parts of the mucosa. Complicated descriptions of the mucosal surface, such as mat or lustreless, are not useful.

To be universally meaningful, any description of mucosal appearances must use only those discontimuous variables which are capable of close definition and which can be consistently reported by observers working independently. Our results 
show that bleeding can be closely defined and described as follows: (1) non-haemorrhagic: no bleeding either spontaneously or to light touch; (2) moderately haemorrhagic: bleeding to light touch, but no spontaneous bleeding seen ahead of instrument at initial inspection ; (3) severely haemorrhagic: spontaneous bleeding seen ahead of instrument at initial inspection with bleeding to light touch (blood on the surface of normal mucosa is thus excluded). Using a similar classification, we, taken in pairs and scoring independently, agreed in more than two out of three patients. There is no final evidence that the degree of haemorrhage from the mucosa is closely related to the severity of the disease, but there is good presumptive evidence that this is so.

Clinical conditions can be graded by summation of the scores of individual criteria as has been done for thyroid disease by Crooks et al. (1959). If a scoring system is not used the definitions for each grade must be mutually exclusive. If this is not so and two unrelated criteria are included in each grade, overlap will often occur so that it becomes impossible to fit some appearances into a single grade. This was one of the causes of failure of the definitions of "activity" used in Patients 1-30, because such a description as "dry, granular, friable" does not correspond to any grade.

\section{Summary}

Three observers recorded independently the sigmoidoscopic appearances of 60 patients with proctocolitis in a study of the observer variation of certain descriptive characteristics.

The observer variation for those characteristics requiring discrimination between poorly defined parts of a continuum, such as shades of colour, largely invalidate their use as comparative terms.

For other characteristics requiring the observer to record the presence or absence of a clearly defined feature, such as spontaneous bleeding and bleeding to light touch, there was less variation, and these assessments are meaningful.

A simple classification of the mucosal appearances in proctocolitis based on these particular characteristics is described.

\section{REFERENCES}

Baron, J. H. Connell, A. M., Lennard-Jones, J. E., and Jones, F. A. 1962). Lancet, 1, 1094

Crooks, J., Murray, I. P. C., and Wayne, E. J. (1959). Quart. F. Med., 28, 211.

Lockhart-Mummery, H. E. (1959). Proc. roy. Soc. Med., 52, Suppl (Anglo-American Conference on Proctology), p. 3.

\title{
Pneumococcal Meningitis in Pregnancy and the Puerperium
}

\author{
ADETOKUNBO O. LUCAS, ${ }^{*}$ M.B., B.SC., M.R.C.P., D.P.H., D.T.M.\&H.
}

Brit. med. F., 1964, 1, 92-95

Pneumococcal meningitis is a disease of world-wide distribution affecting both sexes and all age-groups from neonates to nonagenarians (Ruegsegger, 1942). Although there are isolated reports of this disease occurring in pregnancy and in the puerperium, the literature suggests that such an association is rare and fortuitous (MacQueen, 1927 ; Heinz, 1928 ; Laffont et al., 1933 ; Petersen, 1937 ; Nuckols and Hertig, 1938 ; Hutton 1956; Mahon et al., 1959). In Ibadan, cases of pneurnococcal meningitis in pregnant and recently delivered women are not uncommon. This paper is a report of 26 cases of pneumococcal meningitis in pregnant and puerperal women. Evidence is presented that pregnant and puerperal women in this area are more liable to pneumococcal meningitis than nonpregnant women, and the significance of the finding is discussed.

\section{Material and Methods}

This work is based on the study of all the adult cases with pneumococcal meningitis which were treated at the University College Hospital, Ibadan, between 1 January 1958 and 31 March 1962. I did not see the patients who were admitted before March 1960 at the acute stage of the illness. Data about these were obtained from case notes, although some of the survivors were traced and examined.

The diagnosis in each case was based on examination of the cerebrospinal fluid, which was obtained at necropsy in one patient who died before lumbar puncture could be performed. Stained smears of the fluid were examined and routine bacteriological methods were used for culture. Streptococcus pneu-

\footnotetext{
- Senior Registrar, Department of Medicine, University College Hospital, Ibadan, Nigeria.
}

moniae was identified by the optochin technique (Bowers and Jeffries, 1955). Other investigations on these patients included $x$-ray examination of the chest and paranasal sinuses, blood count and blood culture, and bacteriological examination of sputum or throat swab. Swabs were taken from the vaginal vault in 10 patients.

At an early stage of this investigation it was noted that most of the adult women patients with pneumococcal meningitis were either pregnant or had recently been delivered. In order to determine how far selection of patients was responsible for the high proportion of pregnant and puerperal patients, two other groups of hospital patients were studied: (1) all adult patients who were admitted to this hospital with other types of meningitis (pyogenic, tuberculous, and viral); and (2) all adult patients who were admitted with bacteriologically proved typhoid. Typhoid was chosen for comparison because it is an acute specific fever, commonly encountered in this area, the presenting symptoms being fever, mental confusion, and coma. The period covered was the same as for the pneumococcal meningitis patients. These cases were analysed by age and sex, and whether they were pregnant or recently delivered. A woman was regarded as being recently delivered up to two months after parturition or abortion.

The frequency of pregnant and puerperal cases was also determined in two other groups of patients.

1. Epidemic Meningococcal Meningitis.-In March 1962 a field survey was carried out in Northern Nigeria, where epidemics of meningococcal meningitis recur annually (Horn, 1951). Twenty-three treatment centres were visited and 277 patients were seen.

2. Lobar pneumonia, representing pneumococcal infection without meningitis.-The study was carried out in the general 